Maria Auxiliadora Oliveira 1

Ângela Fernandes Soares do Couto Esher 1

Elizabeth Moreira dos Santos 2

Marly Aparecida Elias Cosendey 1

Vera Lucia Luiza 1,3

Jorge A. Z. Bermudez 1

\section{Avaliação da assistência farmacêutica às pessoas vivendo com HIV/AIDS no Município do Rio de Janeiro}

\author{
Evaluating pharmaceutical services for people \\ living with HIV/AIDS in the city of Rio de Janeiro
}

${ }^{1}$ Núcleo de Assistência Farmacêutica, Departamento de Ciências Biológicas, Escola Nacional de Saúde Pública, Fundação Oswaldo Cruz. Rua Leopoldo Bulhões 1480, Rio de Janeiro, $R J$ 210421-210, Brasil. dora@ensp.fiocruz.br aesher@ensp.fiocruz.br vera@ensp.fiocruz.br bermudez@ensp.fiocruz.br

2 Departamento de Endemias Samuel Pessoa, Escola Nacional de Saúde Pública, Fundação Oswaldo Cruz. Rua Leopoldo Bulhões 1480, Rio de Janeiro, RJ 21041-210, Brasil. bmoreira@ensp.fiocruz.br 3 Centro de Pesquisa Hospital Evandro Chagas, Fundação Oswaldo Cruz. Av. Brasil 4365, Rio de Janeiro, $R J$ 21045-900, Brasil.

\begin{abstract}
This study evaluates access by people living with HIVIAIDS to pharmaceutical care provided by public health care facilities in the city of Rio de Janeiro, Brazil, focusing on availability, accessibility, and accommodation. The evaluation was conducted using the implementation analysis approach, assessing the process of producing intervention outcomes, considering its relationship to the organizational context. A case study was performed in 11 public health facilities selected according to: different health program areas; diverse levels of complexity; and more than 100 HIVIAIDS patients registered. The degree of implementation (89\%) was considered acceptable. Problems with human resources skills and lack of a quality program were identified. Some limitations of the indicators are discussed. Finally, the study highlights the importance of this kind of evaluation as a methodology for continuous monitoring of quality in pharmaceutical care.
\end{abstract}

Key words HIV; Acquired Immunodeficiency Syndrome; Sexually Transmitted Diseases

Resumo Este estudo tem como objetivo avaliar o acesso à assistência farmacêutica, prestada aos portadores do HIVIAIDS, em serviços da rede pública de saúde no Município do Rio de Janeiro, Brasil, focalizando aspectos relacionados às dimensões: disponibilidade, oportunidade e adequação dos recursos. A estratégia utilizada foi a análise de implantação, que permite avaliar os processos envolvidos na produção dos efeitos esperados para uma intervenção, e sua relação com o contexto organizacional. Foi realizado um estudo de caso em onze serviços públicos de saúde, selecionados segundo: representação das diferentes áreas programáticas, nível de complexidade e atenção a mais de cem portadores de HIVIAIDS. O grau de implantação do programa (89\%) foi considerado aceitável. Contudo, foram identificadas deficiências, que podem ser contornadas com treinamento do pessoal envolvido no processo, e com a adoção de um programa da qualidade. São também discutidas algumas limitações dos indicadores utilizados. Concluindo, ressaltase a importância desse tipo de avaliação, como metodologia que permite o monitoramento para a melhoria contínua da qualidade da assistência farmacêutica.

Palavras-chave HIV; Síndrome de Imunodeficiência Adquirida; Doenças Sexualmente Transmissíveis 


\section{Introdução}

O acesso das pessoas vivendo com HIV e AIDS a uma assistência farmacêutica de qualidade representa um dos maiores desafios para os sistemas de saúde, principalmente nos países subdesenvolvidos. O ritmo acelerado da expansão da epidemia nos países pobres tem acarretado uma crescente demanda por cuidados médicos, incluindo o uso de medicamentos anti-retrovirais (ARV), o que impõe aos governantes a necessidade premente de buscar soluções economicamente viáveis para a questão.

A escassez de recursos financeiros, o alto custo dos medicamentos, os reduzidos investimentos em P\&D das indústrias nacionais, o impacto da proteção de patentes de medicamentos, estabelecida no acordo Trade Related Aspects of Intellectual Rights (TRIPS), da Organização Mundial do Comércio, a necessidade de profissionais de saúde treinados são fatores que, na maioria das vezes, inviabilizam ou dificultam o acesso a medicamentos essenciais (Bermudez et al., 2000).

No Brasil, país que possui um programa nacional de controle da infecção pelo HIV/AIDS considerado como um modelo para o mundo, muitas questões estão na ordem do dia, incluindo a qualidade da assistência farmacêutica.

Em 1991, o Ministério da Saúde (MS) implementou uma política de distribuição de medicamentos ARV e para infecções oportunísticas. Até o ano de 1996, havia irregularidade constante no fornecimento desses medicamentos, o que prejudicava a rotina do tratamento da maioria das pessoas vivendo com AIDS. A insuficiência e instabilidade na oferta dos poucos anti-retrovirais disponíveis no mercado, e dos demais medicamentos para o tratamento das infecções oportunísticas, foram os pontos marcantes de uma assistência farmacêutica desprovida de logística eficiente, que onerava estados e municípios (MS, 1999a, 1999b).

Em 13 de novembro de 1996, foi sancionada a Lei 9.313, que garante aos portadores do vírus HIV e AIDS o direito de receber gratuitamente, pelo Sistema Único de Saúde (SUS), todos os medicamentos necessários ao seu tratamento (Brasil, 1996). Essa lei, além de servir como um instrumento legal para reivindicação de direitos, marca o início de grandes mudanças no âmbito da política de assistência farmacêutica aos portadores de HIV/AIDS. Nesse sentido, foi necessário formular e implementar um sistema logístico de medicamentos envolvendo as três esferas de governo, que inclui seleção, programação, aquisição, distribuição e uso racional.
No país, cerca de 90 mil pessoas vivendo com HIV e AIDS recebem os medicamentos ARV na rede pública de saúde. Com gastos crescentes devido ao incremento do número de pacientes em tratamento, ao aumento da proporção de pessoas utilizando terapias mais complexas e à atualização das recomendações para a terapia, o Governo Federal despendeu na compra de medicamentos ARV e outros destinados às principais doenças oportunísticas cerca de US\$ 34 milhões em 1996, US\$ 224 milhões em 1997, US\$ 305 milhões em 1998, US\$ 335 milhões em 1999 e US\$ 303 milhões em 2000. Em relação ao orçamento do MS, a aquisição dos medicamentos para a AIDS correspondeu a $0,24 \%$ em 1996, 1,18\% em 1997, 1,82\% em 1998, 3,18\% em 1999 e estava estimada em $3 \%$ no ano 2000 (MS, 2000, 2001). O acesso universal à terapia ARV, associado ao uso mais difundido de quimioprofilaxia para infecções oportunísticas e a oferta de outros tipos de assistência, têm possibilitado a redução das internações hospitalares e dos óbitos por AIDS no Brasil (MS, 2000). Fonseca \& Barreira (2001) evidenciaram uma diminuição importante na taxa média de crescimento anual da mortalidade por AIDS no Brasil, no período de 1995-1999 $(-12,54 \%)$, em ambos os sexos, mesmo em regiões onde a epidemia apresentou crescimento significativo nos coeficientes de incidência, como é caso do Nordeste, onde a queda da taxa média de mortalidade foi de 7,09\%.

No Estado do Rio de Janeiro, no mesmo período houve uma redução de $48 \%$ no número de óbitos por AIDS (MS, 2000). Segundo Lauria et al. (2000) pode-se observar queda tanto no número absoluto de óbitos quanto na letalidade por AIDS. Adicionalmente, a partir de 1996, ano em que foi iniciada a distribuição dos inibidores de protease em toda a rede municipal de saúde, observa-se um aumento da sobrevida dos portadores do HIV e AIDS.

Segundo a Portaria 176/GM de 8 de março de 1999 (Brasil, 1999), fica sob responsabilidade do MS a aquisição e distribuição dos medicamentos relativos aos Programas Nacionais de AIDS (ARV), Tisiologia, Hanseníase, Sangue e Hemoderivados (Fator VIII, Fator IX, Complexo Protrombínico e DDAVP), Diabetes (Insulina) e Controle de Endemias. A oferta dos medicamentos para o tratamento de doenças oportunísticas em decorrência da infecção pelo HIV fica a cargo das Secretarias Estaduais e Municipais de Saúde.

Com a particularidade de ser o único município do país a receber diretamente do MS os ARV, o Município do Rio de Janeiro estabeleceu uma logística própria para a dispensação de medicamentos a 14.746 pessoas acompanha- 
das nos ambulatórios de 53 unidades, vinculadas a diferentes níveis de gestão: municipal (30), federal (6), estadual (5), universitário (4), filantrópico (4) e militar (4) (SMS, 2000).

Com vistas a fornecer subsídios para o aprimoramento contínuo e, se necessário, a reorientação dos esforços empreendidos pelo Programa, este trabalho tem como objetivo avaliar o acesso à assistência farmacêutica prestada às pessoas vivendo com HIV/AIDS em serviços da rede pública de saúde no município do Rio de Janeiro, focalizando aspectos relacionados às dimensões de disponibilidade, oportunidade e adequação dos recursos (Penchansky \& Thomas, 1981). A estratégia utilizada foi a análise de implantação, que permite avaliar os processos envolvidos na produção dos efeitos esperados para uma intervenção, neste caso, a assistência farmacêutica, em sua relação com o contexto organizacional.

\section{Abordagens conceituais}

\section{Assistência farmacêutica}

O conceito de assistência farmacêutica adotado neste trabalho é aquele definido pela Política Nacional de Medicamentos como: “O grupo de atividades relacionadas com o medicamento destinadas a apoiar as ações de saúde demandadas por uma comunidade. Envolve o abastecimento de medicamentos em todas e em cada uma de suas etapas constitutivas, a conservação e o controle de qualidade, a segurança e a eficácia terapêutica dos medicamentos, o acompanhamento e a avaliação da utilização, a obtenção e a difusão de informação sobre medicamentos e a educação permanente dos profissionais de saúde, do paciente e da comunidade para assegurar o uso racional de medicamentos" (MS, 1999c:34).

Nessa perspectiva, o estabelecimento de uma política nacional de tratamento para pessoas vivendo com HIV/AIDS, deve contemplar todo o conjunto de aspectos compreendidos no conceito de assistência farmacêutica.

Segundo o documento Política de Medicamentos de AIDS do Ministério da Saúde/Brasil, a seleção dos medicamentos, a definição de esquemas terapêuticos, assim como dos critérios clínicos e laboratoriais norteadores do tratamento são estabelecidos pelo MS, por meio de comitês assessores, constituídos por especialistas. No Sistema Logístico de Medicamentos Anti-retrovirais, tanto a programação quanto a aquisição de medicamentos são realizadas pelo MS, com base em um sistema de informação de fluxo ascendente. O processo de distribuição é parcialmente descentralizado, podendo dar-se entre almoxarifados públicos ou diretamente do produtor aos almoxarifados estaduais e municipal (MS, 2000).

Ressalta-se que a Coordenação Nacional de Doenças Sexualmente Transmissíveis/AIDS (CN-DST/AIDS), está desenvolvendo um sistema informatizado, o SICLOM (Sistema Logístico de Controle de Medicamentos) que permite o controle do fluxo de dados e do estoque, fornecendo ainda uma série de informações administrativas e outras relacionadas ao perfil clínico e demográfico da clientela atendida. No entanto, no momento da avaliação, tal sistema ainda não se encontrava em funcionamento em nenhuma das unidades visitadas.

\section{Avaliação}

Dentre as definições existentes para avaliação, neste trabalho, optou-se por aquela proposta por Contandriopoulos et al. (1997:31): “um julgamento de valor a respeito de uma intervenção ou sobre qualquer um de seus componentes, com o objetivo de ajudar na tomada de decisões". Nesta abordagem, a avaliação pretende fornecer informações úteis tanto para responder a problemas enfrentados por gestores, como também para a subsidiar os processos de formulação, implementação e reorientação de políticas.

Uma avaliação deve ser sensível aos contextos político e organizacional, e não pode negligenciar o que foi planejado e executado (os efeitos planejados e os não intencionais). Além disso, ela deve explicitar a orientação teórica que a sustenta. A ausência de uma orientação teórica em um modelo de avaliação, pode resultar em avaliações do tipo "caixa preta", as quais são utilizadas geralmente para analisar os efeitos produzidos por uma determinada intervenção (Chen, 1990). As avaliações do tipo "caixa preta", caracterizam-se por um foco principal na relação global entre os recursos investidos e os resultados obtidos, sem se preocupar com as transformações ocorridas no decorrer da intervenção. A mera análise dos efeitos geralmente estreita os objetivos de um programa, desconsiderando a reflexão sobre a perspectiva dos vários executores e dos diversos meios e contexto de implantação.

A concepção de intervenção é aqui entendida como "um conjunto dos meios (físicos, humanos, financeiros, simbólicos) organizados em um contexto específico, em um dado momento, para produzir bens ou serviços com o objetivo de modificar uma situação problemática" (Contandriopoulos et al., 1997:31). 
Contandriopoulos et al. (1997) destacam dois tipos de avaliação para uma intervenção: avaliação normativa e pesquisa avaliativa. Na avaliação normativa julga-se cada componente da intervenção em relação a normas e critérios existentes. Na pesquisa avaliativa é feito um julgamento ex-post, utilizando procedimento científico para avaliar as relações estabelecidas entre os diferentes componentes da intervenção. A pesquisa avaliativa pode ser composta por seis tipos de análise: (a) análise estratégica, (b) análise da intervenção, (c) análise da produtividade, (d) análise dos efeitos, (e) análise do rendimento e (f) análise da implantação (Contandriopoulos et al., 1997).

A análise da implantação é importante para avaliar intervenções complexas e compostas por elementos seqüenciais, sobre os quais o contexto pode interagir de diferentes modos. Essa análise especifica o conjunto de fatores que influenciam os resultados obtidos após a implementação de uma dada intervenção. Dessa forma, a análise da implantação relacionase diretamente à capacidade de utilizar os resultados das pesquisas avaliativas para tomar decisões sobre generalização de uma intervenção em outros meios.

\section{Acesso a medicamentos}

$\mathrm{O}$ acesso eqüitativo aos serviços e produtos de saúde, tem sido colocado como um dos principais objetivos da política de saúde nos diferentes países, tendo como estratégias fundamentais, por um lado, o aumento da capacidade aquisitiva - sendo um exemplo a política de genéricos - e o conhecimento técnico do consumidor; por outro, o aumento da disponibilidade, aliado à melhor organização dos recursos médicos e dos serviços existentes.

A despeito do conceito de acesso geralmente ser entendido como sinônimo de disponibilidade de serviços e recursos de saúde, deve-se considerar também, a importância de que estes estejam disponíveis no momento e lugar que o paciente necessita, e que a forma de ingresso no sistema seja clara (Aday \& Andersen, 1974). Esses autores são categóricos ao defender que a prova do acesso consiste na utilização de um serviço e não, simplesmente, na existência do mesmo.

Penchansky \& Thomas (1981:128) definem acesso como o "grau de ajuste entre os clientes $e$ o sistema" que estaria representado nas seguintes dimensões:

- disponibilidade (availability), relaciona-se com o volume e o tipo de recursos e serviços oferecidos, e com as necessidades dos clientes;
- oportunidade (accessibility), refere-se à existência dos serviços no lugar e no momento em que é requerido pelos clientes;

- adequação (accomodation), é a maneira como os recursos são organizados para seu fornecimento e a habilidade dos clientes para se adaptar;

- poder aquisitivo (affordability), relacionase ao aspecto do custo, os preços dos serviços e à capacidade de pagamento dos clientes;

- aceitabilidade (acceptability), refere-se às atitudes tanto de provedores quanto dos clientes a respeito de suas caraterísticas e práticas.

Adotamos como definição de acesso a medicamentos essenciais, aquela proposta por Bermudez et al. (1999:13), que, baseado nas considerações anteriores, a propõe como uma "relação entre a necessidade de medicamentos e a oferta dos mesmos, na qual essa necessidade é satisfeita no momento e no lugar requerido pelo paciente (consumidor), com a garantia de qualidade e a informação suficiente para o uso adequado".

$\mathrm{O}$ acesso aos medicamentos ARV é entendido como efeito esperado da assistência farmacêutica aos portadores do HIV/AIDS, embora, neste trabalho, as suas dimensões de disponibilidade, oportunidade e adequação tenham sido utilizadas apenas para orientar a escolha e avaliação dos componentes do programa de medicamentos no nível local, isto é, nas unidades públicas de saúde do Município do Rio de Janeiro.

\section{Material e métodos}

O estudo foi realizado em duas fases. Inicialmente, realizou-se uma pesquisa documental e bibliográfica para a construção do modelo teórico de avaliação, baseado na proposição de Cosendey (2000) (Figura 1). Para isso, foi elaborada uma descrição detalhada da assistência farmacêutica aos portadores do HIV no município do Rio de Janeiro, objetivando a compreensão de cada um dos componentes do programa nos níveis central, regional e local. Essas informações foram reorganizadas, tendo como referências o sistema logístico proposto pela CN-DST/AIDS (MS, 2000), os conceitos de estrutura e processo (Donabedian, 1986) e os critérios de avaliação relativos às dimensões de acesso selecionadas - disponibilidade, oportunidade e adequação (Penchansky \& Thomas, 1981). Esse modelo orientou a escolha dos indicadores (Tabela 1) e a elaboração dos instrumentos de coleta de dados.

Na segunda etapa, foi realizado um estudo de caso no Município do Rio de Janeiro. As unidades de observação selecionadas foram onze 
Modelo teórico de avaliação da assistência farmacêutica. Estrutura e processo.

\begin{tabular}{|l|}
\hline $\begin{array}{l}\text { Nível desencadea- } \\
\text { dor do processo }\end{array}$ \\
\hline $\begin{array}{l}\text { Critérios de } \\
\text { avaliação }\end{array}$ \\
\hline
\end{tabular}

\begin{tabular}{|l|l|l|l|}
\hline Nível central & Nível regional local & $\begin{array}{c}\text { Disponibilidade } \\
\text { Oportunidade } \\
\text { Adequação }\end{array}$ \\
\hline
\end{tabular}
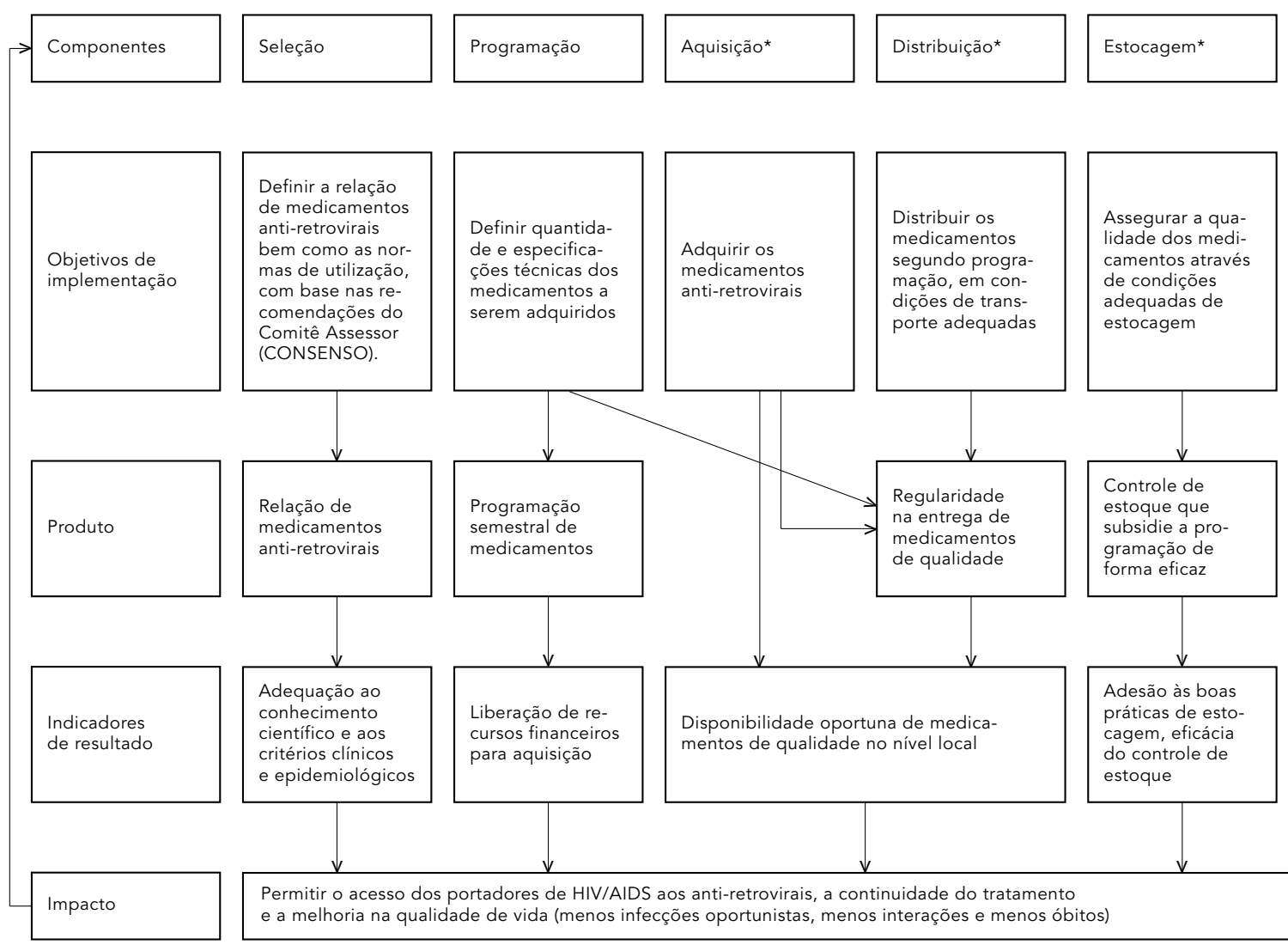

Relação de
medicamentos medicamentos
anti-retrovirais

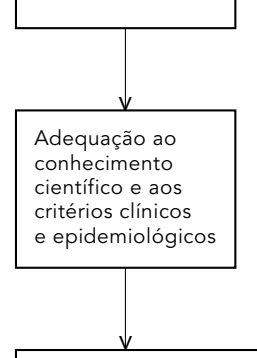

Liberação de recursos financeiros para aquisição

Permitir o acesso dos portadores de HIV/AIDS aos anti-retrovirais, a continuidade do tratamento

e a melhoria na qualidade de vida (menos infecções oportunistas, menos interações e menos óbitos)

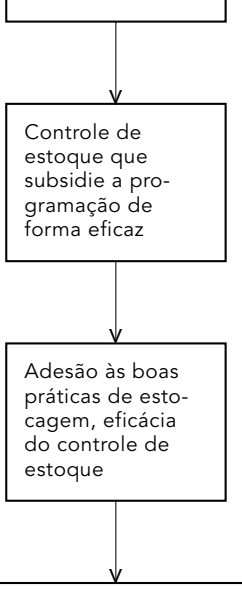

Assegurar a prescrição e dispen-

sação adequadas

junto aos prescri-

tores, dispensado-

res e à população

Disponibilidade oportuna de medica-

mentos de qualidade no nível local
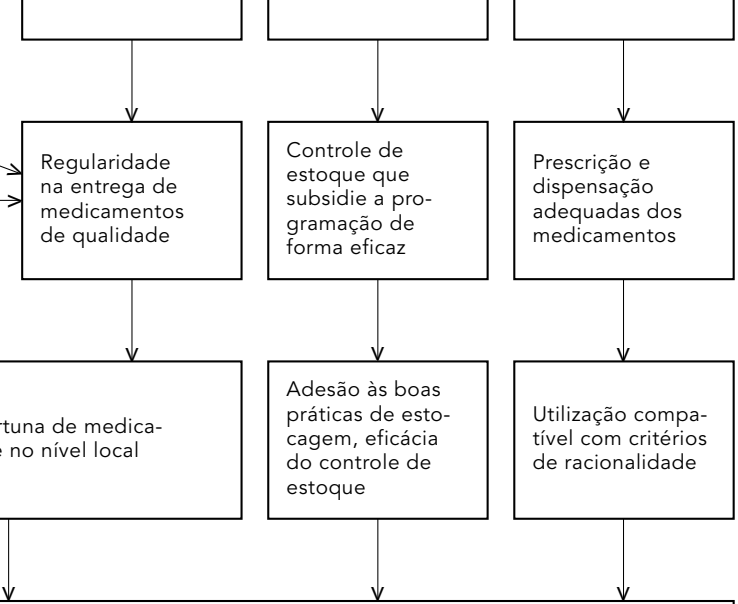

* Componentes do programa avaliados no nível local.

serviços públicos de saúde. Nessa seleção, procurou-se contemplar áreas programáticas que apresentassem diferentes extratos sócio-econômicos e unidades de saúde de complexidade diferenciada (um Hospital Geral, nove Centros de Saúde, um Hospital Universitário), localizados nas áreas programáticas 1, 2, 3, 4 e 5. Além disso, adotou-se como critério de inclusão no estudo, o fato da Unidade de Saúde possuir mais de cem pacientes inscritos no programa de distribuição de ARV.

A metodologia de avaliação privilegiada foi a análise de implantação, que se propõe a iden- tificar os processos envolvidos na produção dos efeitos de uma intervenção, ou seja, especifica o conjunto de fatores que influenciam os resultados obtidos após a introdução da mesma, relacionando componentes internos com o contexto onde é implantada. Assim, mede a influência da variação do grau de implantação da intervenção nos efeitos observados (Denis \& Champagne, 1997).

Os instrumentos construídos para a coleta de dados do projeto foram: (1) questionário estruturado com perguntas fechadas e abertas, (2) formulário para contagem física de medica- 
Tabela 1

Indicadores utilizados.

\begin{tabular}{lll}
$\begin{array}{l}\text { Componente } \\
\text { do programa }\end{array}$ & Denominação & Método de cálculo \\
\hline
\end{tabular}

\section{Estocagem}

B

B 1

Porcentagem de critérios de
BPE observadas em relação à
área de armazenagem.

Aquisição/

C Processo

C 1

Porcentagem média de medicamentos disponíveis em estoque.
Um medicamento é considerado disponível se, ao menos uma unidade do produto, dentro do prazo de validade, encontra-se em estoque. Para o cálculo deste indicador foram contabilizados os medicamentos ARV encontrados no almoxarifado e na farmácia. Esse número foi comparado com a quantidade mensal de ARV fornecida à unidade de cuidado. Essa quantidade mensal foi considerada expressão da necessidade da unidade de saúde no período.

\section{Estocagem}

C 2

C 3

C 4
Porcentagem de medicamentos com prazo de validade vencido.

Porcentagem média ponderada de variação no inventário para os medicamentos estocados.
Porcentagem de medicamentos com prazo de validade vencido, em relação ao total de medicamentos estocados.

Porcentagem média ponderada de variação no inventário é a diferença da porcentagem média ponderada entre os níveis de estoque registrados e a contagem física real.
Porcentagem média do registro

Porcentagem média dos medicamentos
Número de itens de BPE observados dividido pelo número total de itens de BPE analisados (x 100).
$100 \%$

1) Dividir o número de medicamentos em estoque pelo número total de medicamentos ARV programados para a unidade ( $x$ 100);

2) Somar as porcentagens obtidas para cada unidade de saúde e dividir pelo número total de unidades de saúde examinadas.

Número de princípios ativos com prazo de validade vencido sobre o número total de princípios ativos estocados (x 100).

1) Somar o número total de unidades para cada produto como demonstrado no registro;

2) Somar o número total de unidades verificada por contagem física;

3) Subtrair a contagem física da quantidade registrada, retirando o sinal negativo, criando um valor absoluto:

4) Dividir o resultado obtido anteriormente, pela contagem física e multiplicar este quociente por 100 . do estoque que corresponde à contagem física para os em estoque, cuja contagem física insere-se em uma faixa de variação medicamentos ARV em estoque.

Dividir contagem física pelo número total de registros examinados e, multiplicar este resultado por 100 . 


\begin{tabular}{|c|c|c|c|c|}
\hline $\begin{array}{l}\text { Componente } \\
\text { do programa }\end{array}$ & Denominação & Definição & Método de cálculo & Padrão \\
\hline \multicolumn{5}{|l|}{ Dispensação } \\
\hline D 1 & $\begin{array}{l}\text { Porcentagem de adequação } \\
\text { às BPD. }\end{array}$ & $\begin{array}{l}\text { Porcentagem de critérios de BPD } \\
\text { observados. Adequação aos padrões } \\
\text { estabelecidos pela gerência de DST/AIDS } \\
\text { do Município do Rio de Janeiro, para a } \\
\text { dispensação dos medicamentos ARV. }\end{array}$ & $\begin{array}{l}\text { Número de itens de BPD observados } \\
\text { dividido pelo número total de itens de } \\
\text { BPD analisados ( } \times 100) \text {. }\end{array}$ & $100 \%$ \\
\hline D 2 & $\begin{array}{l}\text { Porcentagem de prescrições } \\
\text { adequadas às RTARV-AA de } \\
\text { 1999/MS. }\end{array}$ & $\begin{array}{l}\text { Porcentagem de prescrições classificadas } \\
\text { como aceitáveis segundo as RTARV-AA } \\
\text { de } 1999 / \mathrm{MS} \text {. Nestes documentos } \\
\text { as combinações de tratamento são } \\
\text { classificadas como aceitáveis, não } \\
\text { aceitáveis e de resgate. Neste trabalho, } \\
\text { foram consideradas adequadas as } \\
\text { combinações classificadas como } \\
\text { aceitáveis, a partir da análise da } \\
\text { combinação ARV escrita nas prescrições } \\
\text { médicas, não considerando, portanto, } \\
\text { na análise, dados clínicos ou laboratoriais } \\
\text { dos pacientes. }\end{array}$ & $\begin{array}{l}\text { Número de prescrições de acordo com } \\
\text { a categoria "aceitáveis" dividido pelo } \\
\text { número total de prescrições analizadas } \\
\text { (x 100) }\end{array}$ & $100 \%$ \\
\hline
\end{tabular}

Fonte: MSH/WHO (1997); RPM (1995); WHO (1993).

Legenda: $\mathrm{BPE}=$ Boas práticas de estocagem; $\mathrm{ARV}=$ Anti-retrovirais; $\mathrm{BPD}=$ Boas práticas de Dispensação;

RTARV-AA = Recomendações para terapia anti-retroviral em adultos e adolescentes infectados pelo HIV - 1999.

mentos anti-retrovirais em estoque, (3) lista de verificação para orientar a observação de aspectos importantes da dispensação e (4) formulário para transcrição das receitas ambulatoriais. Os dados referentes às receitas médicas serão analisados em outro trabalho.

Foi elaborado um banco de dados que possibilitou o cálculo dos indicadores estabelecidos (Tabela 1) e do grau de implantação do Programa.

Os componentes estocagem e controle de utilização (Tabela 1), foram representados por indicadores específicos. Porém, as dimensões de aquisição e distribuição foram avaliadas utilizando-se o critério de disponibilidade dos medicamentos no nível local. Dessa forma, um único critério estava representando duas dimensões, motivo pelo qual optou-se por atribuir ponderação diferenciada aos diferentes componentes do Programa, como descrito a seguir: - estocagem: peso 1;

- dispensação: peso 1;

- aquisição/distribuição: peso 2 .

Para a determinação do grau de implantação do Programa, foi utilizado o modelo construído e validado por Cosendey (2000), em um estudo recente que avaliou a implantação do Programa de Farmácia Básica. Esse modelo permitiu contemplar as dimensões propostas no presente projeto, para avaliar o acesso à assistência farmacêutica de pessoas vivendo com HIV e AIDS.
Utilizou-se para a classificação do grau de implantação os seguintes pontos de corte (Cosendey, 2000; Hartz et al., 1997; Ojeda, 1992; OPAS/OMS, 1987):

- aceitável: para o grau de implantação maior ou igual a $80 \%$;

- insatisfatório: para o grau de implantação entre 40 e $79 \%$;

- crítico: para o grau de implantação menor ou igual a $39 \%$.

\section{Resultados e discussão}

Indicadores de processo: aquisição e distribuição, estocagem e controle de utilização

O indicador de processo, utilizado para análise do componente de aquisição e distribuição foi a porcentagem média de medicamentos disponíveis em estoque (C1), que em nove das dez unidades visitadas (uma não tinha registro de estoque), foi de 95\%. Esse indicador apresentou uma limitação na sua interpretação. No momento da visita, todos os frascos de determinadas formulações farmacêuticas já poderiam ter sido dispensados, fazendo com que eles não estivessem disponíveis no almoxarifado ou na farmácia. O controle desse componente, que poderia ter sido realizado padronizando-se o momento da coleta em relação ao 
momento de abastecimento da unidade, não ocorreu. Esse é um aspecto interessante a ser considerado em estudos posteriores que utilizem esse indicador, e incluam dimensões referentes à qualidade do programa.

Os resultados referentes aos indicadores de processo, utilizados para avaliação do componente estocagem são apresentados e discutidos a seguir.

a) A porcentagem de medicamentos com prazo de validade vencido (C2) foi de $2,9 \%$. Informações obtidas nas entrevistas, demonstraram que esse porcentual revela muito pouco da dinâmica das relações entre a unidade local e o almoxarifado de apoio logístico (S/Cincal). Esse setor só aceitava devolução de medicamentos, cujo prazo de validade fosse superior a seis meses. No entanto, o mesmo, eventualmente, distribuía medicamentos com prazo de validade menor que seis meses para as unidades de saúde, ficando estas impedidas de realizar o estorno pela regra anterior. A outra alternativa para evitar que o prazo de validade dos medicamentos expirasse, seria o remanejamento para uma unidade próxima o que, em geral não ocorreu, pois na maioria das vezes, as diferentes unidades dispunham do mesmo lote do medicamento.

Em algumas unidades não se teve acesso à totalidade dos medicamentos vencidos, pois os mesmos estavam estocados em salas não acessíveis. Uma unidade relatou ter meios para destruir seus medicamentos vencidos, em incineradores; outra ainda relatou que "picota seus comprimidos vencidos" e "despeja as soluções na pia”. Esse procedimento expõe o operador e o ambiente a riscos de contaminação, sendo um método inadequado.

b) A porcentagem média ponderada de variação no inventário para os medicamentos estocados (C3), foi de 12,4\%, mostrando uma deficiência no sistema de registro dos medicamentos em estoque. Observou-se ainda, que uma das unidades não possuía registro de estoque. c) A porcentagem média do registro do estoque que corresponde à contagem física para os medicamentos anti-retrovirais em estoque (C4), demonstrou que todas as unidades visitadas encontravam-se fora da variação recomendada, sendo que seis delas se situavam na faixa de 0 a $30 \%$, ou seja, valores considerados muito baixos, sugerindo um controle de estoque ineficaz.

Em relação à avaliação do componente de controle da utilização, um dos indicadores de processo utilizados foi a porcentagem de adequação às boas práticas de dispensação (D1), que envolveu sete descritores: (1) Presença de formulário da Secretaria Municipal de Saúde para avaliação da quantidade de medicamentos anti-retrovirais necessária por mês, (2) Freqüência de preenchimento do formulário, (3) Período de envio do formulário, (4) Formas de utilização do formulário, (5) Fluxo correto de envio do formulário, (6) Disponibilidade de medicamentos para aqueles que necessitam iniciar o tratamento imediatamente e (7) Disponibilidade de medicamentos para aqueles que necessitam trocar o esquema terapêutico imediatamente. A porcentagem obtida a partir dos dados coletados em entrevistas com os responsáveis por esta atividade, foi de $77,3 \%$. A observação local revelou um quadro ainda menos favorável, pois algumas respostas afirmativas não foram confirmadas, como por exemplo, a orientação ao paciente no momento da dispensação, que não foi constatada em nenhuma das unidades visitadas. $\mathrm{O}$ argumento mais freqüente para a pouca ou nenhuma orientação ao paciente no momento da dispensação foi a possível exposição do indivíduo, que poderia vir a ser identificado na fila como portador do HIV. A orientação ao paciente fica, segundo esses profissionais, sob responsabilidade exclusiva dos médicos durante as consultas. Em muitos serviços, foram encontrados funcionários administrativos, desviados de função e sem qualquer formação específica, dispensando medicamentos para os clientes do Programa de AIDS.

Considerando a importância da orientação ao paciente no ato da dispensação, deve-se buscar alternativas que permitam viabilizá-la preservando a privacidade do paciente, pois se por um lado, esse momento é extremamente importante para complementar as informações fornecidas pelo médico, por outro, é visível o constrangimento daqueles que estão na fila para receber os medicamentos anti-retrovirais. Os vários frascos, grandes ou pequenos, são recebidos pelos usuários em sacos plásticos com cores (não-transparentes), fornecidos por eles próprios ou pela farmácia, sendo ilustrativo dessa situação.

$\mathrm{O}$ atendimento às receitas de origem da rede privada é um procedimento que se dá de forma diferenciada entre as unidades. Foram encontradas unidades que não atendem essas receitas, outras que obrigam o cliente a passar pelo médico da unidade para que este transcreva a receita, outras atendem receitas de médicos cadastrados na unidade. Entretanto, todas exigem o cadastramento do cliente no programa de AIDS da unidade, conforme o preconizado.

É importante ressaltar que, os anti-retrovirais não foram objeto de controle especial na 
maioria das unidades, mesmo fazendo parte do elenco de medicamentos sujeitos a controle especial, conforme a Portaria no 344 (Brasil, 1998).

Muitos dos problemas encontrados nas farmácias das unidades, foram justificados pelas constantes mudanças de coordenadores ou gerentes. Profissionais treinados são transferidos ou desviados de suas funções e não repassam as informações para seus sucessores, dificultando o processo de implantação do programa.

Nas unidades onde não havia funcionários envolvidos prioritariamente ou exclusivamente no programa de AIDS, os responsáveis afirmaram que todos estavam habilitados a dispensar os medicamentos anti-retrovirais.

Não existia previsão de uma cota adicional de medicamentos para inclusão de novos pacientes nem para a mudança de regime terapêutico. Essa situação era parcialmente contornada pelo uso de medicamentos de pacientes faltosos ou doados por usuários que trocam de esquema terapêutico, abandonam o tratamento ou falecem. Em todas as unidades havia muitos medicamentos doados, cujos frascos na maioria das vezes estavam abertos, o que claramente compromete a qualidade dos mesmos.

O outro indicador utilizado para avaliar o componente de controle da utilização, foi a porcentagem de prescrições classificadas como aceitáveis, segundo as Recomendações para Te- rapia Anti-retroviral em Adultos e Adolescentes infectados pelo HIV (RTARV-AA) de 1999/MS (D2), que foi de $91 \%$.

\section{Indicador de estrutura: estocagem}

O indicador de estrutura utilizado para análise do componente estocagem foi a porcentagem de critérios de boas práticas de estocagem observados (B1), que revelou quatro unidades utilizando, simultaneamente, os espaços do almoxarifado e da farmácia para estocagem dos medicamentos anti-retrovirais. As demais - na maioria das vezes, Centros Municipais de Saúde - estocavam em apenas um dos espaços ou não os tinham bem definidos. Em média, as unidades visitadas cumpriam apenas cerca de $51 \%$ dos critérios recomendados. Almoxarifados sujos, caixas de medicamentos em contato com piso e paredes, deficiência no número de geladeiras para estocagem do medicamento Ritonavir e falta de termômetros e de registros de temperatura (de geladeira e ambiente), foram os aspectos mais freqüentemente observados na avaliação.

O consolidado dos indicadores trabalhados pode ser observado na Figura 2.

No cômputo geral dos indicadores, o cálculo do grau de implantação do programa foi de $89 \%$, sendo considerado aceitável, relacionando-se de forma coerente à redução de mortali-

Figura 2

Resultados observados para os indicadores utilizados, em valores percentuais médios e padrões de referência. Rio de Janeiro, Brasil, dezembro de 1999 a abril de 2000.

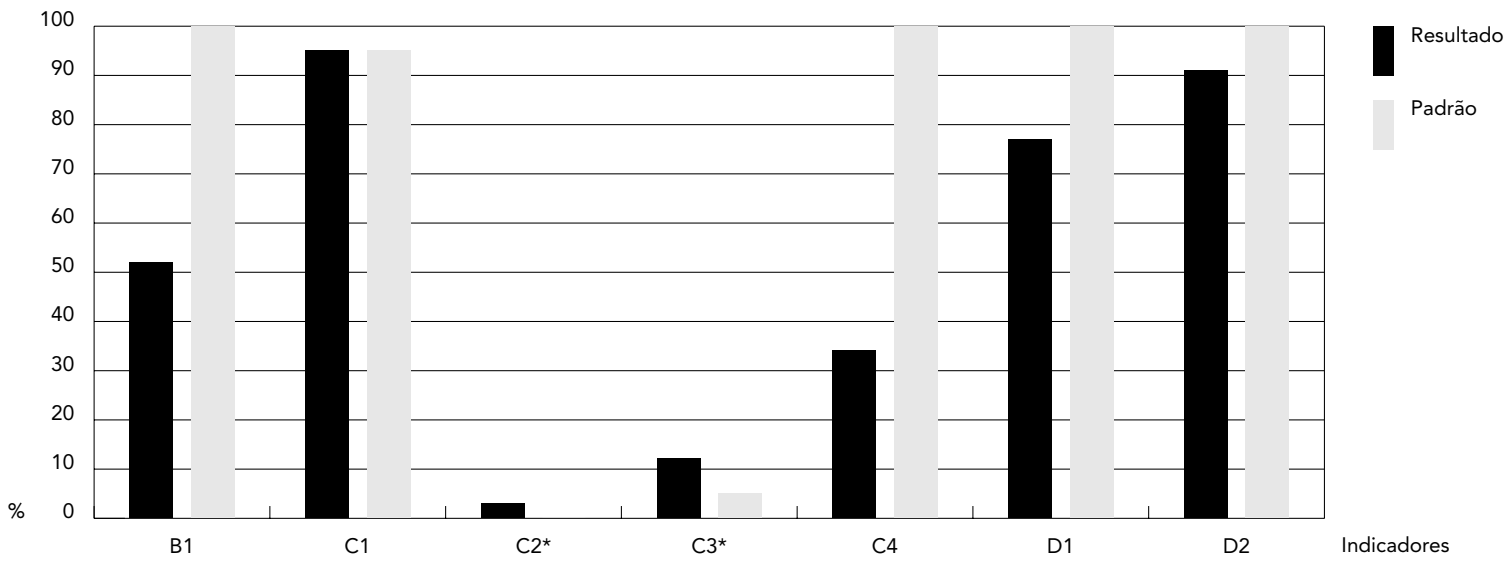

* Nestes indicadores o valor desejado é igual ou menor ao padrão; nos demais o valor desejado é igual ou maior ao padrão. 
dade observada e discutida na introdução deste trabalho.

\section{Considerações finais}

Os indicadores de estrutura e processo, utilizados para avaliar os componentes da estocagem e do controle da utilização, possibilitaram a verificação do ambiente e das práticas de estocagem dos medicamentos, além dos procedimentos seguidos na dispensação e na prescrição de medicamentos anti-retrovirais.

O valor bastante favorável de $94,7 \%$ para o indicador de disponibilidade, é certamente o mais expressivo para mostrar o sucesso do programa, sendo um dos fatores que contribuem para os efeitos observados na redução das taxas de internação, da mortalidade específica e da incidência de infecções oportunísticas. A despeito do grau de implantação aceitável, alguns indicadores apontam para a necessidade de implementar medidas de intervenção, no sentido de aumentar a efetividade e eficiência do programa.

É, por exemplo, preocupante o fato de algumas unidades não apresentarem registros atualizados da movimentação de estoque, procedimento este que possibilita o acompanhamento contínuo do fluxo de medicamentos movimentados pela farmácia. $\mathrm{O}$ alto custo dos anti-retrovirais e a necessidade de utilização regular dos medicamentos, torna essa prática ainda mais importante.

Um outro aspecto importante que deve ser ressaltado é a existência de medicamentos vencidos dentro e fora do estoque. Um dos fatores que contribuem para essa situação é a relatada obrigatoriedade imposta pelo S/Cincal, do recebimento de um pacote fechado de medicamentos, dentre os quais, alguns eventualmente se encontram com o prazo de validade próximo ao vencimento. A esse respeito existem ainda orientações contraditórias por parte do Programa de DST/AIDS do município e do S/Cincal.

Em relação à capacitação de recursos humanos, seria recomendável a inclusão mais sistemática de todos os profissionais envolvidos com o atendimento a esses pacientes, inclusive os de nível médio. Ainda mais, se considerado que os resultados do estudo demonstraram a existência de problemas em áreas onde o conhecimento técnico é bem estabelecido e documentado na literatura clássica, como é o caso das boas práticas de estocagem ou da existência de medicamentos vencidos no estoque disponível para a dispensação. Uma outra medida que traria bons resultados, seria a implantação de programas de qualidade nas farmácias da rede, com foco no planejamento de ações e no desenvolvimento de programas de avaliação permanente, interna e externa.

Ressalta-se ainda, a importância da implantação do SICLOM que, uma vez funcionando conforme o planejado, certamente permitirá uma agilização e melhor controle de todo o processo logístico dos medicamentos, reduzindo a carga burocrática e promovendo a racionalização do trabalho.

Essas medidas certamente trarão benefícios, não somente aos clientes do programa de AIDS, mas ao conjunto dos pacientes atendidos nessas unidades, na medida em que, a melhoria da qualidade do programa de AIDS certamente terá reflexos nas demais atividades da assistência farmacêutica.

Concluindo, ressalta-se ainda a importância desse tipo de estratégia de avaliação, enquanto metodologia testada e validada, que permite o monitoramento para a melhoria contínua da qualidade da assistência farmacêutica.

\section{Agradecimentos}

Os autores agradecem o apoio financeiro recebido da Fundação Oswaldo Cruz - Programa de Apoio à Pesquisa Estratégica em Saúde (PAPES II) e Convênio FIOCRUZ/FAPERJ para Pesquisador Visitante -, e logístico propiciado pela Secretaria Municipal de Saúde do Rio de Janeiro, particularmente pela Gerência de Doenças Sexualmente Transmissíveis e Aids, cujos técnicos Dra. Betina Durovni, Dra. Valéria Saraceni e Dr. Sérgio Aquino, viabilizaram o acesso aos serviços de saúde selecionados. 


\section{Referências}

ADAY, L. \& ANDERSEN, R., 1992. Marco teórico para el estudio del acceso a la atención médica. In: Investigaciones sobre Servicios de Salud (K. L. White, org.), pp. 604-613, Washington, DC: Organización Panamericana de la Salud.

BERMUDEZ, J. A. Z.; EPSZTEJN, R.; OLIVEIRA, M. A. \& HASENCLEVER, L., 2000. O Acordo Trips da OMC e a Proteção Patentária no Brasil: Mudanças Recentes e Implicações para a Produção Local e Acesso da População aos Medicamentos. Rio de Janeiro: Escola Nacional de Saúde Pública, Fundação Oswaldo Cruz/Organização Mundial da Saúde.

BERMUDEZ, J. A. Z.; LUIZA, V. L.; HARTZ, Z. M. A.; MOSEGUI, G. B. G.; OLIVEIRA, M. A.; COSENDEY, M. A. E. \& ROMERO, C. N. P., 1999. Avaliação do Acesso aos Medicamentos Essenciais: Modelo Lógico e Estudo Piloto no Estado do Rio de Janeiro, Brasil. Rio de Janeiro: Escola Nacional de Saúde Pública, Fundação Oswaldo Cruz. (mimeo.)

BRASIL, 1996. Lei № 9.313 de 13 de Novembro de 1996. Dispõe sobre a Distribuição Gratuita de Medicamentos aos Portadores do HIV e Doentes de AIDS. 27 Dezembro $2000<$ http://www.aids.gov.br/ assistencia/lei9313.htm>.

BRASIL, 1998. Portaria no 344 de 12 de maio de 1998. Aprova o Regulamento Técnico sobre Substâncias e Medicamentos Sujeitos a Controle Especial. Regulamentação de Medicamentos Sujeitos a Controle Especial (Psicoativos e Outros). 30 Julho 2001 <http://www.saude.gov.br/Portarias/Portaria 344-98>.

BRASIL, 1999. Portaria no 176/GM de 8 de março de 1999. Estabelece critérios e requisitos para a qualificação dos municípios e estados ao incentivo à Assistência Farmacêutica Básica e define valores a serem transferidos. Brasília: Diário Oficial da República Federativa do Brasil, no 47E, p. 22, 11 mar, Seção I.

CHEN, H. T., 1990. Method and Theory in Program Evaluation: A Question of Balance. Theory-Driven Evaluations. Beverly Hills: Sage Publications.

CONTANDRIOPOULOS, A. P.; CHAMPAGNE, F.; DENIS, J. L. \& PINEAULT, R., 1997. A avaliação na área da saúde: Conceitos e métodos. In: Avaliação em Saúde: Dos Modelos Conceituais à Prática na Análise da Implantação de Programas (Z. M. A. Hartz, org.), pp. 29-48, Rio de Janeiro: Editora Fiocruz.

COSENDEY, M. A. E., 2000. Análise da Implantação do Programa Farmácia Básica: Um Estudo Multicêntrico em Cinco Estados do Brasil. Tese de Doutorado, Rio de Janeiro: Escola Nacional de Saúde Pública, Fundação Oswaldo Cruz.

DENIS, J. L. \& CHAMPAGNE, F., 1997. Análise de implantação. In: Avaliação em Saúde: Dos Modelos Conceituais à Prática na Análise da Implantação de Programas (Z. M. A. Hartz, org.), pp. 49-88, Rio de Janeiro: Editora Fiocruz.

DONABEDIAN, A., 1986. Criteria and standards for quality assessment and monitoring. $Q R B, 12: 99-108$.

FONSECA, M. G. P. \& BARREIRA, D., 2001. A Evolução da Mortalidade por AIDS no País, Segundo Distribuição Geográfica. 3 Agosto 2001 <http://www. aids.gov.br/sitebol/>.
HARTZ, Z. M. A.; CHAMPAGNE, F.; CONTANDRIOPOULOS, A. P. \& LEAL, M. C., 1997. Avaliação do programa materno-infantil: Análise de implantação em sistemas locais de saúde no Nordeste do Brasil. In: Avaliação em Saúde: Dos Modelos Conceituais à Prática na Análise da Implantação de Programas (Z. M. A. Hartz, org.), pp. 89-131, Rio de Janeiro: Editora Fiocruz.

LAURIA, L. M.; YIAGO, S.; PACHECO, A.; CRUZ, M.; AQUINO, S.; SARACENI, V. \& DUROVNI, B., 2000. A mortalidade por AIDS no município do Rio de Janeiro. In: I Fórum e II Conferência de Cooperação Técnica Horizontal da América Latina e do Caribe em HIV/AIDS e DST, Resumos, v. I, p. 292. Brasília: Ministério da Saúde.

MS (Ministério da Saúde), 1999a. Terapia Anti-retroviral e Saúde Pública - Um Balanço da Experiência Brasileira. Brasília: MS.

MS (Ministério da Saúde), 1999b. Controle do HIV/ AIDS: A Experiência Brasileira. Brasília: MS.

MS (Ministério da Saúde), 1999c. Política Nacional de Medicamentos/Secretaria de Políticas de Saúde. Brasília: MS.

MS (Ministério da Saúde), 2000. Política de Medicamentos de AIDS do Ministério da Saúde/Brasil. 27 Dezembro $2000<$ http://www.aids.gov.br/assistencia/politica_medic_aids_brasil.htm>.

MS (Ministério da Saúde), 2001. Acesso Universal e Gratuito. 13 Março 2001 <http://www.aids.gov.br/ politica/Acesso\%20Universal\%20e\%20Gratuito/ acesso.htm>.

MSH (Management Sciences for Health)/WHO (World Health Organization), 1997. Managing Drug Supply: The Selection, Procurement, Distribution, and Use of Pharmaceuticals. West Hartford: Kumarian Press.

OJEDA, N. S., 1992. Evaluación de servicios de salud maternoinfantil en América Latina. Foro Mundial de la Salud, 13:140-143.

OPAS (Organização Pan-Americana da Saúde)/OMS (Organização Mundial da Saúde), 1987. Condições de Eficiência dos Serviços de Atenção Materno Infantil. Washington, DC: OPAS.

PENCHANSKY, D. \& THOMAS, J., 1981. The concept of access: Definition and relationship to consumer satisfaction. Medical Care, 20:127-140.

RPM (Rational Pharmaceutical Management Project), 1995. Rapid Pharmaceutical Management Assessment: An Indicator-Based Approach. Washington, DC: Pan American Health Organization.

SMS (Secretaria Municipal de Saúde), 2000. DST/ AIDS 2000 - Boletim Epidemiológico. Saúde em Foco, 9 (20).

WHO (World Health Organization), 1993. How to Investigate Drug Use in Health Facilities: Select Drug Use Indicators. Geneva: WHO.

Recebido em 8 de outubro de 2001

Versão final reapresentada em 8 de fevereiro de 2002 Aprovado em 26 de abril de 2002 